\title{
Doble evaluación de la susceptibilidad por movimientos en masa basada en redes neuronales artificiales y pesos de evidencia
}

\author{
Paul Goyes-Peñafiel ${ }^{1 *}$; Alejandra Hernandez-Rojas $^{2}$
}

Forma de citar: Goyes-Peñafiel, P.; Hernandez-Rojas, A. (2021). Doble evaluación de la susceptibilidad por movimientos en masa basada en redes neuronales artificiales y pesos de evidencia. Boletín de Geología, 43(1), 173-191. https://doi.org/10.18273/revbol.v43n1-2021009

Material Suplementario: Este artículo tiene Material Suplementario con las figuras de todos los factores condicionantes derivados del ráster y de tipo vectorial.

\section{Resumen}

Los movimientos en masa son la amenaza natural más frecuente en zonas tropicales y son causantes de graves daños en la infraestructura vial, pérdida de vidas humanas y consecuencias económicas. Por lo tanto, una evaluación cuantitativa y confiable de la susceptibilidad por movimientos en masa es esencial para el desarrollo y planeación territorial. En este trabajo se estudia el cálculo de la susceptibilidad con un bajo nivel de incertidumbre a partir de la integración metodológica de Pesos de Evidencia con las Redes Neuronales Artificiales. El primero se usó para extraer los valores de los pesos de las variables y el inventario de movimientos en masa, y el segundo para establecer una relación no lineal entre los factores condicionantes y el inventario puntual de movimientos en masa realizado a partir del estudio geológico y geomorfológico del municipio de Popayán. Esto genera una doble verificación que permite extraer las características de las variables categóricas y continuas para generar relaciones más precisas de susceptibilidad, evitando la multicolinealidad y el uso de factores no significantes a partir del Análisis de Componentes Principales. Para estudiar la influencia de las variables, se analizaron dos propuestas metodológicas, la primera con dos variables y la segunda con cinco variables explicativas. Para cada una de ellas se aplicaron los métodos cuantitativos de Regresión Logística, Perceptrón Multicapa y Red Neuronal Profunda como elementos de doble verificación. Los resultados fueron evaluados a partir de la curva Característica Operativa del Receptor para cada modelo, encontrando que las redes neuronales profundas tienen un valor de Área Bajo la Curva de 0,902 y 0,969 para las propuestas 1 y 2 respectivamente, superando al método de Pesos de Evidencia usado convencionalmente y a la Regresión Logística como métodos cuantitativos.

Palabras clave: Susceptibilidad por movimientos en masa; Aprendizaje Profundo; Regresión Logística; Pesos de Evidencia; Análisis de Componentes Principales; Redes Neuronales Artificiales.

\section{Double landslide susceptibility assessment based on artificial neural networks and weights of evidence}

\begin{abstract}
.
Landslides are the most frequent natural hazards in tropical regions. They cause serious damages to road infrastructure, human losses and effects on economy. Therefore, a quantitative and reliable evaluation of landslide susceptibility is important for territorial planning and development. In this work, the susceptibility calculation is studied with a low uncertain level through the methodological integration of Weights of Evidence method with Artificial Neural Networks. The first one was used to extract the weighted values from the association of variables and the landslide inventory, and the second one to establish the non-linear relation between the conditioning factors and the punctual landslide inventory obtained through the geologic and geomorphologic study of the Popayan municipality. This produces a double verification allowing to extract the characteristics of categorical and continuous variables to produce more accurate susceptibility relations, avoiding multicollinearity and non-significant factors through the Principal Component Analysis. For studying the influence of variables, two methodological proposals were analyzed, the first one with two variables and the second one with five explanatory variables. For each one, it was applied Logistic Regression, Multilayer Perceptron, and Deep Neural Network quantitative methods as elements of double verification. The results of each model were assessed by the Receiver Operating Characteristics curves. The Deep Neural Networks got an Area Under the Curve with values of 0.902 and 0.969 for proposals 1 and 2, respectively, overcoming Weights of Evidence and Logistic Regression as quantitative methods.
\end{abstract}

Keywords: Landslide Susceptibility; Deep Learning; Logistic Regression; Weights of Evidence; Principal Component Analysis; Artificial Neural Networks.

\footnotetext{
${ }^{1}$ Escuela de Ingeniería de Sistemas e Informática, Universidad Industrial de Santander, Bucaramanga, Colombia. (*) yesid.goyes@correo.uis.edu.co

${ }^{2}$ Escuela de Geología, Universidad Industrial de Santander, Bucaramanga, Colombia. maria.hernandez26@correo.uis.edu.co
} 


\section{Introducción}

Los movimientos en masa son considerados como una de las amenazas naturales más comunes y catastróficas, responsables de pérdidas humanas y de efectos negativos sobre el medio ambiente físico (Wang et al., 2019). El acelerado aumento de la población urbana en las últimas décadas ocupando terrenos susceptibles a fenómenos de origen natural ha generado un aumento considerable en el riesgo asociado con movimientos en masa (Aristizábal et al., 2019). Por esta razón, el desarrollo de metodologías y herramientas que permitan calcular e identificar las áreas susceptibles a estos eventos de una manera económica y eficiente, resulta de interés para las entidades territoriales al momento de ejecutar acciones para la prevención y mitigación de los daños potenciales (Bragagnolo et al., 2020). En el caso de Colombia, con el Decreto 1077 de 2015, el Gobierno Nacional ha impulsado la expedición de políticas y estrategias de gestión orientadas a establecer consideraciones técnicas a tener en cuenta para la ejecución de estudios relacionados a identificar las áreas susceptibles por movimientos en masa (Servicio Geológico Colombiano, 2017).

Los mapas de susceptibilidad por movimientos en masa describen la distribución espacial de la probabilidad de ocurrencia de estos eventos en un área, con base en factores geológicos y ambientales (Dai et al., 2002). Estos factores pueden ser de naturaleza categórica (vectorial) como las unidades geológicas superficiales, la geomorfología, y los usos del suelo; o de naturaleza continua como los ráster derivados del Modelo Digital de Elevaciones - MDE (Servicio Geológico Colombiano, 2017). Los métodos utilizados para el cálculo de estos mapas, a escala regional, se pueden agrupar en heurísticos y estadísticos (Corominas et al., 2014). Los métodos heurísticos son enfoques directos en los que un experto evalúa la susceptibilidad por movimientos en masa directamente en campo, o indirectos cuando, con base en el juicio experto, se asigna un peso a cada parámetro considerado significante para la ocurrencia por movimientos en masa (Lin et al., 2017; Luo y Liu, 2018; van Westen et al., 2008). Por otro lado, los métodos estadísticos cuantifican la susceptibilidad con base en el inventario de deslizamientos y los factores condicionantes (Luo y Liu, 2018; Trigila et al., 2015). Estos métodos incluyen los modelos estadísticos bivariados como: Pesos de Evidencia y Relación de Frecuencia (Aditian et al., 2018; Hong et al., 2017; Mahdadi et al., 2018; Pamela et al., 2018) o multivariados como la Regresión Logística (Cantarino et al., 2019; Hemasinghe et al., 2018; Kadavi et al., 2019; Lombardo y Mai, 2018; Mahdadi et al., 2018; Oh et al., 2018; Yang et al., 2019; Zhao et al., 2019). Sin embargo, estas metodologías no aprovechan al máximo la naturaleza categórica o continua de los factores condicionantes (variables explicativas-evidenciales), ya que requiere la conversión de datos continuos a discretos o viceversa, induciendo así un cambio en los valores intrínsecos de las variables condicionantes. Por lo tanto, la integración adecuada de estos datos representa uno de los desafíos más importantes en el cálculo de la susceptibilidad por movimientos en masa.

Este trabajo tiene como objetivo presentar una estrategia de doble evaluación para el cálculo de la susceptibilidad por movimientos en masa. La doble evaluación se analiza primero aplicando el método Pesos de Evidencia para extraer la relación evidencial entre las variables categóricas y el inventario de polígonos con movimientos en masa. En segundo lugar, se aplica Regresión Logística y Redes Neuronales Artificiales (Perceptrón Multicapa y Redes Neuronales Profundas) para estudiar la relación no lineal entre las variables evidenciales obtenidas con Pesos de Evidencia y un inventario puntual de deslizamientos como datos de entrenamiento del modelo. Una aplicación de la metodología presentada se evalúa y compara en el municipio de Popayán con base en los métodos de Pesos de Evidencia y Regresión Logística, Aprendizaje de Máquina (Perceptrón Multicapa) y Redes Neuronales Profundas (Aprendizaje Profundo). Estos tres últimos métodos conforman algunos de los algoritmos que permiten resolver el Problema de la Clasificación, lo que en estadística consiste en construir modelos de clasificación que permitan predecir información a partir de datos de entrenamiento (Bui et al., 2020; Tharwat, 2020). Finalmente, los resultados obtenidos fueron validados mediante los valores de Área Bajo la Curva (AUC, por sus siglas en inglés) de la Curva Característica Operativa del Receptor (ROC, por sus siglas en inglés), los cuales están basados en la sensibilidad (fracción de verdaderos positivos) y en 1 - especificidad (fracción de falsos negativos) (Bui et al., 2020, 2016).

\section{Marco teórico}

\section{Regresión Logística}

La Regresión Logística (RL) es un método estadístico cuantitativo usado ampliamente en los análisis de susceptibilidad por movimientos en masa (Yang et al., 2019; Zhao et al., 2019). La aplicabilidad de este método está dada por la capacidad de calcular la 
probabilidad de la presencia de deslizamientos como una función de variables o factores condicionantes (Sagar et al., 2018). En la siguiente ecuación se presenta la formulación de la Regresión Logística como una función de probabilidad de ocurrencia:

$$
\begin{aligned}
\log \left(\frac{p}{1-p}\right) & =b_{0}+\sum_{i=1}^{n} b_{i} \cdot x_{i}=Z, \\
p & =\frac{1}{1+e^{-Z^{\prime}}}
\end{aligned}
$$

donde $p$ es la probabilidad de ocurrencia, $x_{i}$ son las variables independientes analizadas, $b_{0}$ es el intercepto y $b_{i}$ son los coeficientes de la Regresión Logística, los cuales se interpretan como los pesos de las variables independientes.

Las variables pueden ser continuas, discretas, o incluso una combinación de ambas; la principal ventaja radica en que el método de Regresión Logística no necesita que dichas variables tengan una distribución normal (Mahdadi et al., 2018). No obstante, se debe garantizar que las variables sean independientes entre sí, o que tengan una multicolinealidad con tendencia a cero. La multicolinealidad existe cuando se evidencia una alta correlación entre las variables analizadas, lo que puede afectar negativamente en la interpretación de los coeficientes de la regresión (Lin et al., 2017; Patriche et al., 2016). A pesar de ser uno de los algoritmos más usados debido a su fácil implementación, la Regresión Logística no puede resolver problemas de manera eficiente donde la superficie de decisión sea no lineal. En la práctica, esto implica que la región de decisión para los datos binarios se hace usando un medio plano limitado por un hiperplano (Goodfellow et al., 2016), lo que no permite analizar otros aspectos más complejos de la naturaleza que abarcan los movimientos en masa. Esta desventaja se puede superar usando algoritmos que involucren más estructuras no lineales, como lo son el Perceptrón Multicapa, cuya región de decisión corresponde a regiones cerradas o conexas; y Aprendizaje Profundo, con regiones de decisión de complejidad arbitraria limitada por el número de neuronas (Correa et al., 2016).

\section{Aprendizaje Profundo}

En los análisis cuantitativos para los mapas de susceptibilidad por movimientos en masa, las Redes Neuronales Artificiales han estado relacionadas con la capacidad de predecir o calcular la probabilidad de producirse un evento dado un conjunto de factores condicionantes (Zhou et al., 2018). Los principales tipos de Redes Neuronales Artificiales aplicados a este campo y, según desde su complejidad, pueden ir desde un Perceptrón Simple (Figura 1a), Perceptrón Multicapa (MLP por sus siglas en inglés) (Figura 1b) y Redes Neuronales Profundas (DNN por sus siglas en inglés) (Figura 1c). En Goodfellow et al. (2016) se desarrollan los aspectos matemáticos y computacionales de las Redes Neuronales Artificiales usadas en este estudio.

Un caso intuitivo del Perceptrón Simple es la Regresión Logística (ecuación 1), la cual es un caso particular del perceptrón donde el vector de entradas es $x_{0}=[\mathrm{A}, \mathrm{B}]^{\mathrm{T}} \in \mathbb{R}^{2 \times 1}$, y la capa de salida $C$ estará definida por $x_{1}=\Theta_{2}\left(W_{1} \cdot x_{0}+b_{1}\right)$, con el vector $W_{1} \in \mathbb{R}^{1 \times 2}$ con los pesos y la función de activación sigmoide $\Theta_{2}(\cdot)$ mostrada en la ecuación 2:

$$
\Theta_{2}(x)=\frac{1}{1+e^{-x}}
$$

En la Figura 1b se incluye una capa oculta, donde la definición de los pesos es $\mathrm{W}_{1} \in \mathbb{R}^{3 \times 2} \mathrm{y}_{2} \in \mathbb{R}^{1 \times 3}$. A este tipo de red se le conoce como Red Neuronal Artificial (ANN por sus siglas en inglés) y, a pesar de su simplicidad, ha constituido una alternativa importante debido a su agilidad, precisión y efectividad, por lo cual MLP representa una nueva generación de métodos cuantitativos para calcular el índice de susceptibilidad (Abbaszadeh-Shahri et al., 2019; Aditian et al., 2018; Bragagnolo et al., 2020; Hu et al., 2020). No obstante, debido a recientes avances en la generación de nuevos algoritmos de retropropagación más eficientes, y con la definición de funciones de pérdida más coherentes con el problema de la susceptibilidad por movimientos en masa, es posible mejorar la exactitud de las predicciones por medio de las DNN, donde se pueden integrar varias capas ocultas con un número variable de neuronas. En la Figura 1c se muestra una DNN de arquitectura 2-103-10-1. En este trabajo se usa la función de pérdida tipo entropía binaria cruzada (binary crossentropy) mostrada en la siguiente ecuación:

$$
L(\mathrm{y}, \hat{\mathrm{y}})=-\frac{1}{N} \sum_{i=0}^{N}\left[y_{i} \log \left(\hat{\mathrm{y}}_{l}\right)+\left(1-y_{i}\right) \log \left(1-\hat{\mathrm{y}}_{i}\right)\right],
$$

donde $y_{i}$ es el valor de la etiqueta de entrenamiento, $\hat{y}_{l}$ es el dato estimado y $N$ es el número de datos en el conjunto de entrenamiento.

Esta función mide qué tan lejos del valor verdadero (que es 0 o 1) se encuentra la predicción para cada una de las clases, y luego promedia estos errores de clase para obtener la pérdida final. Esta función de pérdida es principalmente usada en decisiones binarias 
(Chollet, 2018), lo cual se ajusta bien a la naturaleza del problema de predicción de deslizamientos, donde la etiqueta en los datos de entrenamiento contiene valores de 0 (sin deslizamiento) y 1 (con deslizamiento).

Adicionalmente, en este trabajo se usa la función de activación sigmoide para la capa de salida, debido a que esta función representa la probabilidad de ocurrencia del evento analizado. Para las capas ocultas se utiliza la función de activación Unidad Lineal Rectificada mostrada en la ecuación 4 (ReLU, por sus siglas en inglés), ya que ha mostrado un alto desempeño en otros trabajos del estado del arte relacionados con susceptibilidad por movimientos en masa (Bui et al., 2020; Huang et al., 2020).

$$
\Theta_{1}(x)=\max (0, x)
$$

a)

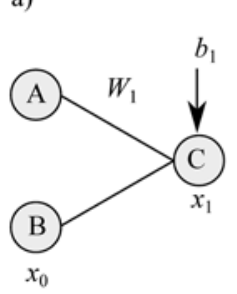

b)
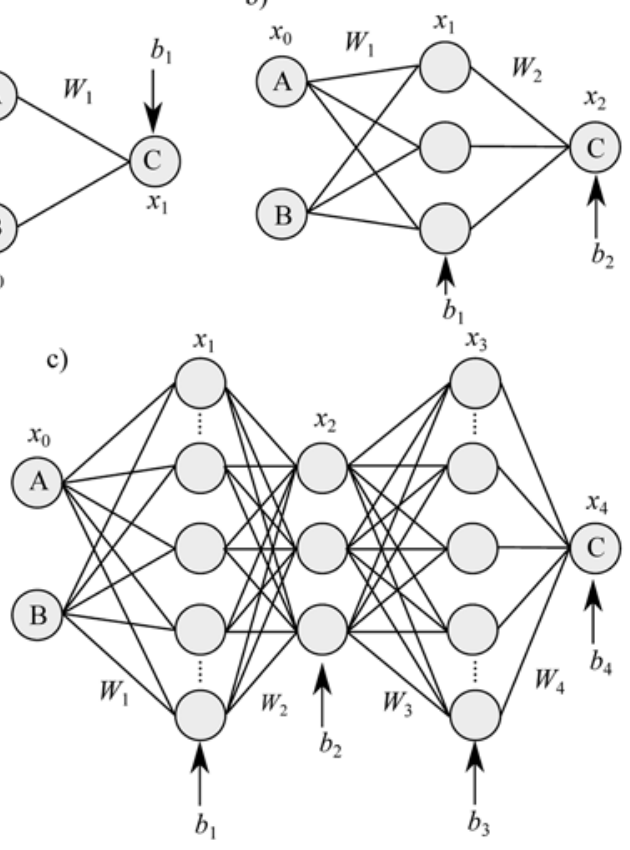

Finalmente, la retropropagación (backpropagation) se realiza con el optimizador Adam, un algoritmo basado en el gradiente de primer orden de funciones objetivo estocásticas y estimaciones adaptativas de momentos de orden inferior. Este algoritmo demostró una manera eficiente de resolver el problema de optimización de grandes volúmenes de datos en comparación con los otros algoritmos basados en el gradiente descendiente (Brownlee, 2017; Kingma y Ba, 2015). Finalmente, una formulación directa de DNN (Figura 1c) queda descrita como:

$$
\begin{aligned}
& x_{1}=\Theta_{1}\left(W_{1} \cdot x_{0}+b_{1}\right), W_{1} \in \mathbb{R}^{10 \times 2} \text { y } x_{0} \in \mathbb{R}^{2 \times 1} \text {, } \\
& x_{2}=\Theta_{1}\left(W_{2} \cdot x_{1}+b_{2}\right), W_{2} \in \mathbb{R}^{3 \times 10} \text { y } x_{1} \in \mathbb{R}^{10 \times 1} \text {, } \\
& x_{3}=\Theta_{1}\left(W_{3} \cdot x_{2}+b_{3}\right), W_{3} \in \mathbb{R}^{10 \times 3} \text { y } x_{2} \in \mathbb{R}^{3 \times 1} \text {, } \\
& x_{4}=\Theta_{2}\left(W_{4} \cdot x_{3}+b_{4}\right), W_{4} \in \mathbb{R}^{1 \times 10} \text { y }_{3} \in \mathbb{R}^{10 \times 1} \text {, }
\end{aligned}
$$

Figura 1. Representación de la arquitectura de (a) un Perceptrón Simple 2-1, (b) Perceptrón Multicapa (MLP) 2-3-1 y (c) Red Neuronal Profunda (DNN) 2-10-3-10-1.

\section{Pesos de Evidencia}

Pesos de Evidencia (WoE por sus siglas en inglés) hace parte de los métodos cuantitativos para el estudio y cálculo de la susceptibilidad por movimientos en masa, además, es un método basado en datos (data-driven). WoE está sustentado en el teorema de Bayes y en los conceptos de probabilidad conocida y posterior (Pamela et al., 2018; Regmi et al., 2010). A través de su implementación, es posible calcular la importancia relativa de los factores condicionantes con un significado estadístico (Ilia y Tsangaratos, 2016). Para la susceptibilidad por movimientos en masa, este método tiene como objetivo estimar, dado un conjunto de especificaciones (factores condicionantes), la estabilidad o inestabilidad de ciertas regiones analizadas (variables dependientes). Esto se calcula mediante la relación y distribución espacial de áreas conocidas y afectadas por deslizamientos, y la distribución de los factores condicionantes divididos o clasificados en rangos que forman una variable nominal o categórica (Ilia et al., 2010; Ilia y Tsangaratos, 2016; van Westen et al., 2003). 
En este método los pesos positivos $\left(W_{i}^{+}\right)$y negativos $\left(W_{i}^{-}\right)$son asignados a cada una de las diferentes clases dentro de un factor condicionante, por ejemplo, a cada unidad o subunidad geomorfológica que hacen parte del mapa de subunidades geomorfológicas. Ilia y Tsangaratos (2016) desarrollan una explicación detallada del WoE. En la siguiente ecuación se presenta una breve formulación del WoE a partir del teorema de Bayes (van Westen, 2002; van Westen et al., 2003):

$$
\begin{aligned}
& W_{1}^{+}=\log \left(\frac{P\left\{B_{i} \mid S\right\}}{P\left\{B_{i} \mid \bar{S}\right\}}\right), \\
& W_{1}^{-}=\log \left(\frac{P\left\{\bar{B}_{l} \mid S\right\}}{P\left\{\bar{B}_{l} \mid \bar{S}\right\}}\right),
\end{aligned}
$$

donde $B_{i}$ y $\bar{B}_{l}$ es la presencia o ausencia del factor condicionante respectivamente. $S$ y $\bar{S}$ representan la presencia o ausencia de deslizamientos en dichos factores condicionantes.

Una vez obtenidos los pesos de evidencia de la ecuación 6 , se define que el peso final evidencial es igual a $W_{f}=W_{i}^{+}+W_{i}^{-}$. Este valor es interpretado de la siguiente forma: (a) $W_{f}=0$ cuando no existe relación evidencial entre el inventario de movimientos en masa y el factor condicionante, (b) $W_{f}>0$ o asociación positiva cuando la presencia del factor condicionante contribuye a la ocurrencia del fenómeno evidenciado en el inventario, y (c) $W_{f}<0$ o asociación negativa cuando la presencia del factor condicionante no contribuye a la ocurrencia del fenómeno evidenciado en el inventario.

\section{Metodología}

Determinar la combinación óptima de factores hace parte inicial en la construcción del modelo de susceptibilidad por movimientos en masa (He et al., 2019; Ozdemir y Altural, 2013; Wang et al., 2019). Con el objetivo de aplicar, analizar y estudiar la predicción de la susceptibilidad por movimientos en masa con WoE y cada uno de los siguientes modelos de Redes Neuronales Artificiales: Perceptrón Simple, Perceptrón Multicapa y Redes Neuronales Profundas, se utilizó el municipio de Popayán como caso de estudio. Este municipio cuenta con estudios previos y un detallado inventario de movimientos en masa y cartografía geológica superficial (Servicio Geológico Colombiano, 2015).

\section{Área de estudio e inventario de movimientos en masa}

El municipio de Popayán, capital del departamento del Cauca, está localizado en el Valle Superior Interandino del Río Cauca, limitado por el flanco occidental de la Cordillera Central y en flanco oriental de la Cordillera Occidental (Figura 2). Presenta una altitud promedio de 1985 msnm y comprende un área total municipal de 478,3 $\mathrm{km}^{2}$ y un área urbana de $41,7 \mathrm{~km}^{2}$ con una población estimada de 270000 habitantes (DANE, 2019).

Desde un enfoque geomorfológico y geológico, el municipio de Popayán presenta tres zonas con características diferenciables, lo que permite hacer un análisis de aplicación de la susceptibilidad con doble evaluación propuesta en este trabajo, donde es posible comparar la susceptibilidad calculada y el inventario o zonas geomorfológicamente activas de deslizamientos. Hacia el sector oriental se presentan geoformas altamente susceptibles a movimientos en masa debido al relieve escarpado y montañoso, con pendientes que alcanzan los $45^{\circ}$ de inclinación asociadas a geoformas de origen volcánico de la Cordillera Central. Las rocas en este sector corresponden a cuerpos ígneos plutónicos y volcánicos, aunque en algunas zonas, debido a los procesos de meteorización, se presentan suelos residuales. Similar a esta área, al occidente del municipio se presenta una alta densidad de movimientos en masa como producto del relieve colinado y de los procesos de meteorización que genera suelos residuales poco cohesivos. Estas dos zonas permiten identificar áreas susceptibles a movimientos en masa en el municipio de Popayán previo a una evaluación. Por otra parte, el casco urbano y el centro del municipio corresponden a una planicie topográfica con pendientes inferiores a los $10^{\circ}$ (ver Figura S4), donde se encuentra un amplio abanico volcánico consecuencia de la acumulación de material piroclástico transportado por canales de flujo (Servicio Geológico Colombiano, 2015). A diferencia de las dos primeras zonas, la susceptibilidad por movimientos en masa en la parte central del municipio es baja, lo cual se corrobora con la ausencia de eventos en esta área (ver Figura S1 y S2).

El inventario de movimientos en masa fue proporcionado por el Servicio Geológico Colombiano (2015) y está basado en 816 eventos definidos a 
través del trabajo de campo y en la interpretación de imágenes satelitales de Google Earth, y fue recopilado en un catálogo histórico del Sistema de Información de Movimientos en Masa-SIMMA en la página de internet del SGC (www.simma.sgc.gov.co). Los movimientos en masa dentro del área de estudio están localizados principalmente en suelos residuales con pendientes entre $10^{\circ}-25^{\circ}(82 \%)$, y en menor proporción en unidades de roca (18\%). Su extensión varía de 39428 $\mathrm{m}^{2}$ a $26 \mathrm{~m}^{2}$, con un área promedio de $2264 \mathrm{~m}^{2}$. De acuerdo con el tipo de movimiento, los movimientos en masa fueron clasificados según Varnes (1978) en deslizamientos $(72,8 \%)$, caídas $(10 \%)$, complejos $(0,2 \%)$, de extensión lateral $(0,1 \%)$ e indefinidos $(16,8 \%)$. A su vez, las caídas se subdividieron según el tipo de material en caída de tierra $(91,5 \%)$, caída de detritos $(6,1 \%)$ y caída de rocas $(2,4 \%)$. Además de los 816 polígonos de movimientos en masa utilizados para el análisis con WoE, fueron creados 400 puntos para el entrenamiento y validación de los modelos de Redes Neuronales Artificiales (Perceptrón Simple, Perceptrón Multicapa, Aprendizaje Profundo), los cuales se clasificaron según su susceptibilidad a la ocurrencia de movimientos en masa en 0 (probabilidad baja de movimiento en masa) y 1 (probabilidad alta de movimiento en masa). Los puntos con probabilidad baja se clasificaron con base en la información geológica del área de estudio (ver Material Suplementario), mientras que los puntos con probabilidad alta corresponden a los centroides de algunos de los polígonos del inventario de movimientos en masa.

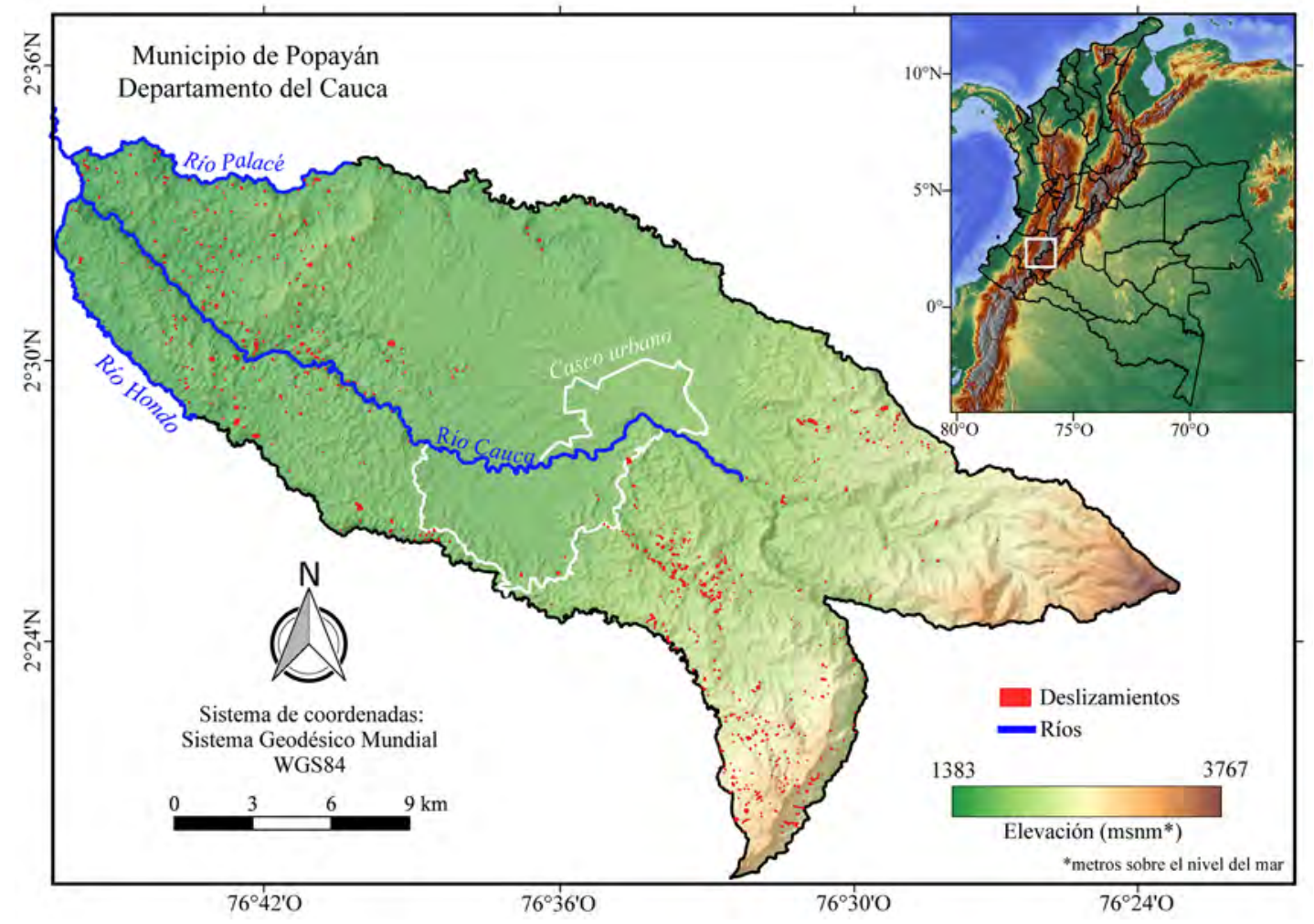

Figura 2. Localización del área de estudio y del inventario de deslizamientos.

\section{Factores condicionantes}

Teniendo en cuenta las condiciones geológicas y ambientales del área de estudio, se escogieron 11 variables relacionadas con la ocurrencia de movimientos en masa según el Decreto 1077 de 2015 y el Servicio Geológico Colombiano (2017) para el cálculo de la susceptibilidad (Tabla 1). Entre las variables categóricas se incluyen las unidades geológicas superficiales $\left(V_{1}\right)$, la geomorfología $\left(V_{2}\right)$ y los usos del suelo $\left(V_{3}\right)$. Para las variables continuas, se escogieron aquellas derivadas del MDE ALOS PALSAR (Asf-Alaska, 2007) con una resolución de $12,5 \mathrm{~m}$ : pendientes $\left(X_{1}\right)$, índice de rugosidad del terreno $\left(X_{2}\right)$, índice topográfico del terreno $\left(X_{3}\right)$, curvatura de plano $\left(X_{4}\right)$, curvatura de perfil $\left(X_{5}\right)$, curvatura general $\left(X_{6}\right)$, longitud del flujo $\left(X_{7}\right)$ y acumulación del flujo $\left(X_{g}\right)$. 
A continuación se presenta una definición de cada variable referente a su aporte a la susceptibilidad: Las unidades geológicas superficiales (Figura S1) representan el material expuesto en el terreno con características físicas que lo hacen más o menos susceptible a la ocurrencia de movimientos en masa (Servicio Geológico Colombiano, 2017). La geomorfología (Figura S2) está relacionada con los procesos geológicos y meteóricos que han modelado el terreno (Carvajal-Perico, 2012). Los usos del suelo (Figura S3) muestran las condiciones actuales de vegetación y de actividad humada en el territorio (van Westen et al., 2008).

Tabla 1. Nombres y tipos de variables usadas en este trabajo. Información recopilada del Servicio Geológico Colombiano (simma.sgc.gov.co).

\begin{tabular}{cccc}
\hline Variable & Nombre & Tipo de dato & $\begin{array}{c}\text { Tipo de } \\
\text { variable }\end{array}$ \\
\hline Y & Puntos de entrenamiento y validación & Punto & Binario \\
$P$ & Inventario de movimientos en masa & Polígono & Constante \\
$V_{1}$ & Unidades Geológicas Superficiales & Polígono & Categórica \\
$V_{2}$ & Geomorfología & Polígono & Categórica \\
$V_{3}$ & Usos del suelo & Polígono & Categórica \\
$X_{1}$ & Pendientes & Ráster & Continua \\
$X_{2}$ & Índice de Rugosidad del Terreno & Ráster & Continua \\
$X_{3}$ & Índice Topográfico de Humedad & Ráster & Continua \\
$X_{4}$ & Curvatura de plano & Ráster & Continua \\
$X_{5}$ & Curvatura de perfil & Ráster & Continua \\
$X_{6}$ & Curvatura general & Ráster & Continua \\
$X_{7}$ & Longitud del flujo & Ráster & Continua \\
$X_{8}$ & Acumulación del flujo & Ráster & Continua \\
\hline
\end{tabular}

La pendiente controla la velocidad del movimiento del material ante un evento de deslizamiento (AbbaszadehShahri et al., 2019; Trigila et al., 2015; Zhao et al., 2019). El índice de rugosidad del terreno (Figura S5) indica la ondulación o la variación del relieve (Servicio Geológico Colombiano, 2015). El índice de humedad del terreno (Figura S6) es usado para caracterizar la distribución espacial de la humedad en el suelo y el volumen de escorrentía (He et al., 2019). Las curvaturas de componente plano (Figura S7), perfil (Figura S8) y general (Figura S9) son parámetros morfométricos que representan la variación espacial del gradiente de la pendiente y resaltan la convergencia (curvatura cóncava) o divergencia (curvatura convexa) de los flujos de agua (Trigila et al., 2015). La longitud del flujo (Figura S10) es la distancia más larga del cauce del flujo hasta la división del drenaje (Regmi et al., 2010). Finalmente, la acumulación del flujo (Figura S11) es el número de celdas aguas arriba que fluyen hacia cada celda del área de estudio (Trigila et al., 2015).

\section{Estrategia evaluativa}

La metodología y estrategia evaluativa consistió en la elaboración de dos propuestas que permiten estimar la precisión de los diferentes modelos cuantitativos usados para el análisis de la susceptibilidad por movimientos en masa. Para las 8 variables derivadas del MDE se aplicó un preprocesamiento utilizando el Análisis de Componentes Principales (PCA por sus siglas en inglés), el cual permite hallar los principales espacios ortogonales en los que se pueden proyectar los componentes de un grupo de datos, y garantiza que, debido a su ortogonalidad, se evite la multicolinealidad y se reduzca la dimensionalidad sin perder la información que describe un sistema (Baeza y Corominas, 2001; Correa-Muñoz e Higidio-Castro, 2017; Gwelo, 2019; Lei et al., 2011). Para este estudio se eligieron los dos primeros componentes principales y en adelante se mencionarán como PC1 y PC2, cuya varianza explicada acumulada fue $68,59 \%$. Como los componentes principales son variables continuas, se clasificaron en 5 cuantiles, y de menor a mayor cuantil se les asignó una variable nominal de 1 hasta 5 .

La estrategia de doble evaluación consiste en el uso del método WoE para estimar las zonas con mayor susceptibilidad evidencial a generar movimientos en masa. En esta etapa se utilizan las variables PC1, PC2, $\mathrm{V}_{1}, \mathrm{~V}_{2}, \mathrm{~V}_{3}$, y el inventario de deslizamientos en polígonos $P$ como se muestra en la Figura 3 a. De esta forma, se 
calculan los pesos de evidencia según la ecuación 6 y finalmente se estima el valor $W_{f}=W_{i}^{+}+W_{i}^{-}$para cada una de las 5 variables (nombradas en adelante K1, K2, K3, K4 y K5). Posteriormente, se utiliza el inventario de 400 puntos evidenciales $Y$ con la información de ocurrencia $(Y=1)$ o ausencia $(Y=0)$, cuyos valores están directamente relacionados con la susceptibilidad por movimientos en masa. De esta forma MLP y DNN se entrenan utilizando las variables de entrada K1, K2, $\mathrm{K} 3$, K4 y K5, y los puntos evidenciales $Y$ como valores de entrenamiento. Adicionalmente, se presentan dos propuestas comparativas para las cuales se valuaron la capacidad predictiva de la susceptibilidad por movimientos en masa:

Propuesta 1: se reducen las variables K1, K2, K3, K4, $\mathrm{K} 5$ a dos variables A, B siguiendo lo mostrado en la Figura 3 b. Posteriormente, se calcula la susceptibilidad por movimientos en masa usando los métodos cuantitativos RL, MLP y DNN, con A y B como variables de entrada para cada uno respectivamente (Figura 4a).

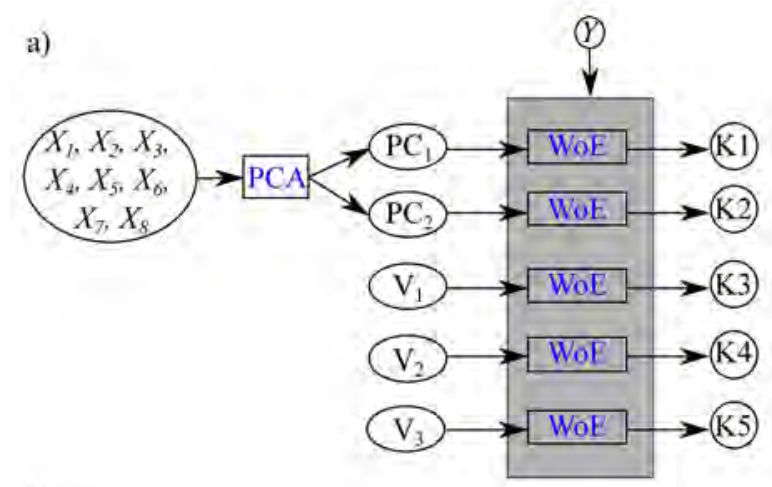

b)
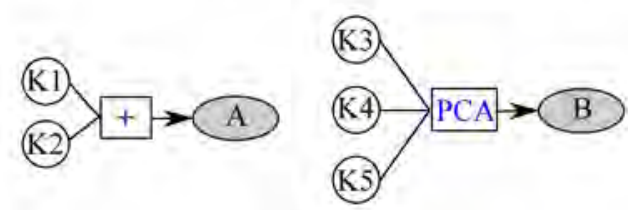

Figura 3. Esquema general para el cálculo de las variables usadas en los métodos RL, MLP y DNN. (a) Aplicación del método WoE sobre los factores condicionantes analizados. (b) Propuesta 1 con la reducción a dos variables usando el Análisis de Componentes Principales.

Propuesta 2: se utilizan las variables K1, K2, K3, K4, K5 como variables de entrada y se aplican los modelos RL, MLP y DNN (Figura 4b).

Para los modelos de MLP, DNN y RL se utilizó el $75 \%$ de los datos para entrenamiento y el $25 \%$ para validación, seleccionados de manera aleatoria. La verificación de la eficacia en la predicción se analizó según las curvas ROC y su respectivo valor de AUC.

a)
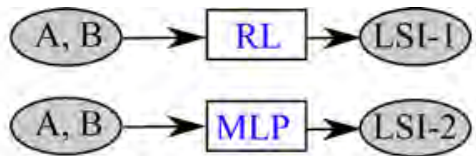

b)

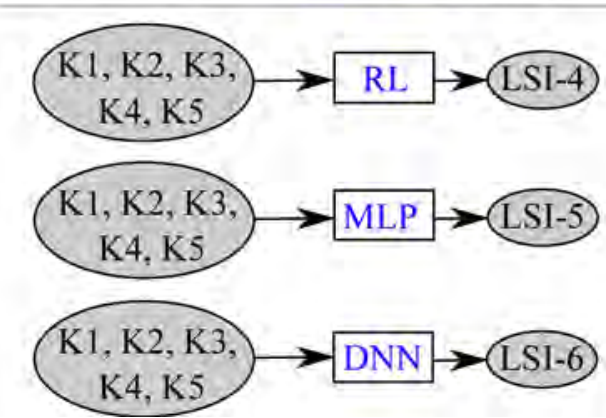

Figura 4. Esquema general para el cálculo del Índice de Susceptibilidad por Movimientos en Masa (LSI) y comparación entre RL, MLP y DNN. (a) Propuesta 1 con la reducción a dos $\mathrm{A}$ y $\mathrm{B}$ variables usando el Análisis de Componentes Principales y (b) Propuesta 2 con el uso de 5 variables evidenciales $\mathrm{K} 1, \mathrm{~K} 2, \mathrm{~K} 3, \mathrm{~K} 4, \mathrm{~K} 5$.

\section{Resultados}

En la Figura 5 se muestra la matriz de dispersiones para las variables K1, K2, K3, K4, K5. Se evidencia la baja correlación entre variables, lo que concuerda con el valor absoluto del coeficiente de correlación de Pearson siempre por debajo de 0,57. Lo anterior, por lo tanto, garantiza que se evita la colinealidad de las variables. Los resultados presentados para MLP y DNN son los que mejor rendimiento computacional y precisión mostraron, y finalmente se decidió por el uso de las arquitecturas mostradas en la Figura 1b y $1 \mathrm{c}$ respectivamente. Con una tasa de aprendizaje de 0,001 y un número de épocas asignado de 500 y 100 para MLP y DNN, respectivamente.

Para la Propuesta 1 se presentan los modelos de clasificación y curvas de validación en la Figura 6. Para RL se observa una frontera de decisión lineal de los puntos analizados en la Figura 6a, los coeficientes de la regresión muestran un ajuste donde todas las variables son significantes, se comprueba una desviación estándar baja y $|\mathbf{z}|>2$ (test de Wald), por lo tanto, cumple que la probabilidad de los datos insignificantes es $\mathbf{p}(>|\mathbf{z}|)<0,05$ (ver Tabla 2). Para MLP la clasificación o frontera de decisión es lineal en los valores positivos y oblicua para valores negativos como se muestra en la Figura 6b, lo que genera un 
ajuste más ambiguo. En la aplicación de la arquitectura DNN (Figura 6c) se obtuvo una frontera de decisión bilineal, es decir, con dos pendientes diferentes, donde se presenta una inclinación mayor para datos negativos. Adicionalmente, el modelo DNN es más robusto que MLP, debido a que se puede verificar una convergencia del modelo para un número de épocas menor (100 en DNN y 500 en MLP); y a partir de sus curvas de validación y entrenamiento, se verifica que para los modelos entrenados no existe sobreajuste (overfitting).
Finalmente, en la Figura 6d se observa la evaluación de cada modelo a partir de los valores de AUC de sus respectivas curvas ROC, donde se obtuvo 0,898, 0,901 y 0,902 para RL, MLP y DNN, respectivamente. La predicción con menos exactitud corresponde a RL, mientras que MLP y DNN apenas tienen una diferencia de $\mathrm{AUC}=0,00104$ por lo que son modelos equivalentes de ajustes para este tipo de datos.

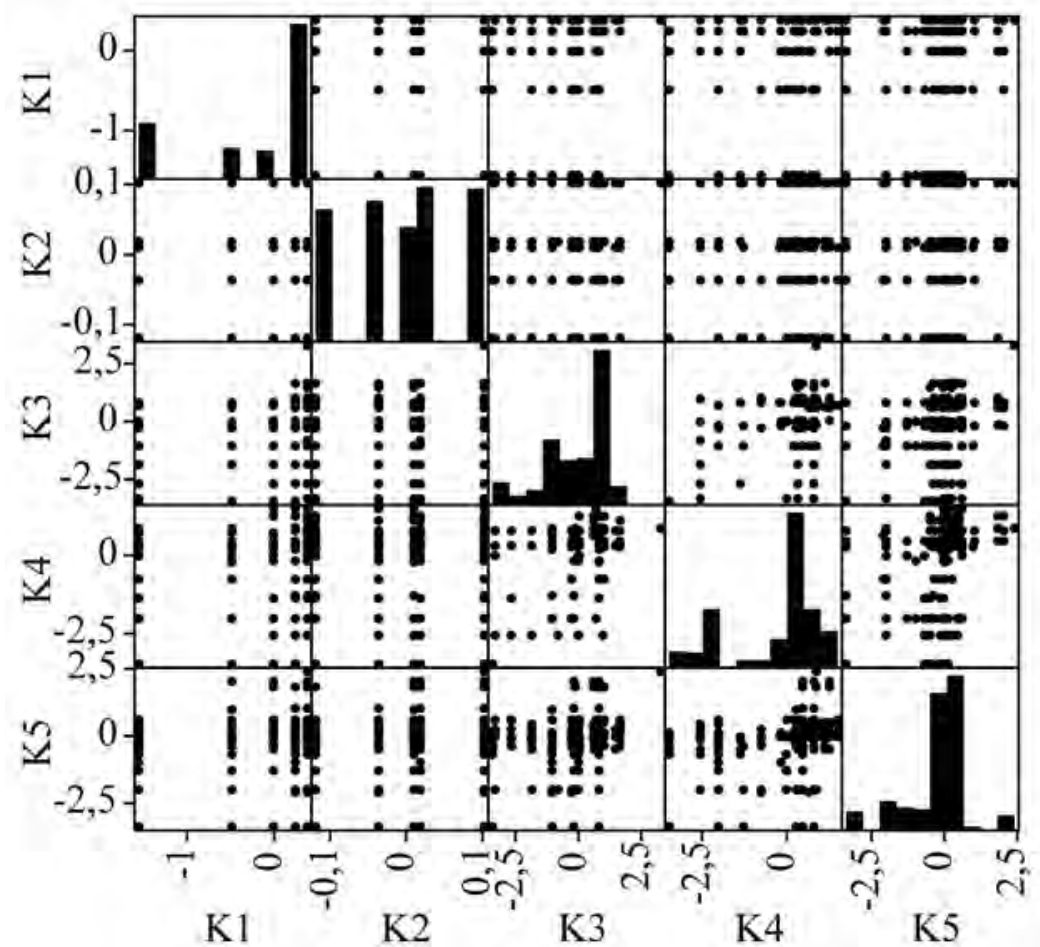

Figura 5. Matriz de dispersión para las variables K1, K2, K3, K4, K5. En la diagonal se muestra el histograma para cada variable.

Tabla 2. Resumen estadístico de RL para la Propuesta 1 con las variables A y B.

\begin{tabular}{ccccc}
\hline & Coeficiente & Desviación estándar & $\mathbf{z}$ & $\mathbf{p}(>|\mathbf{z}|)$ \\
\hline A & $-0,813$ & 0,121 & $-6,706$ & 0,0000 \\
B & 0,948 & 0,116 & 8,158 & 0,0000 \\
Intercepto & 1,149 & 0,159 & 7,232 & 0,0000 \\
\hline
\end{tabular}



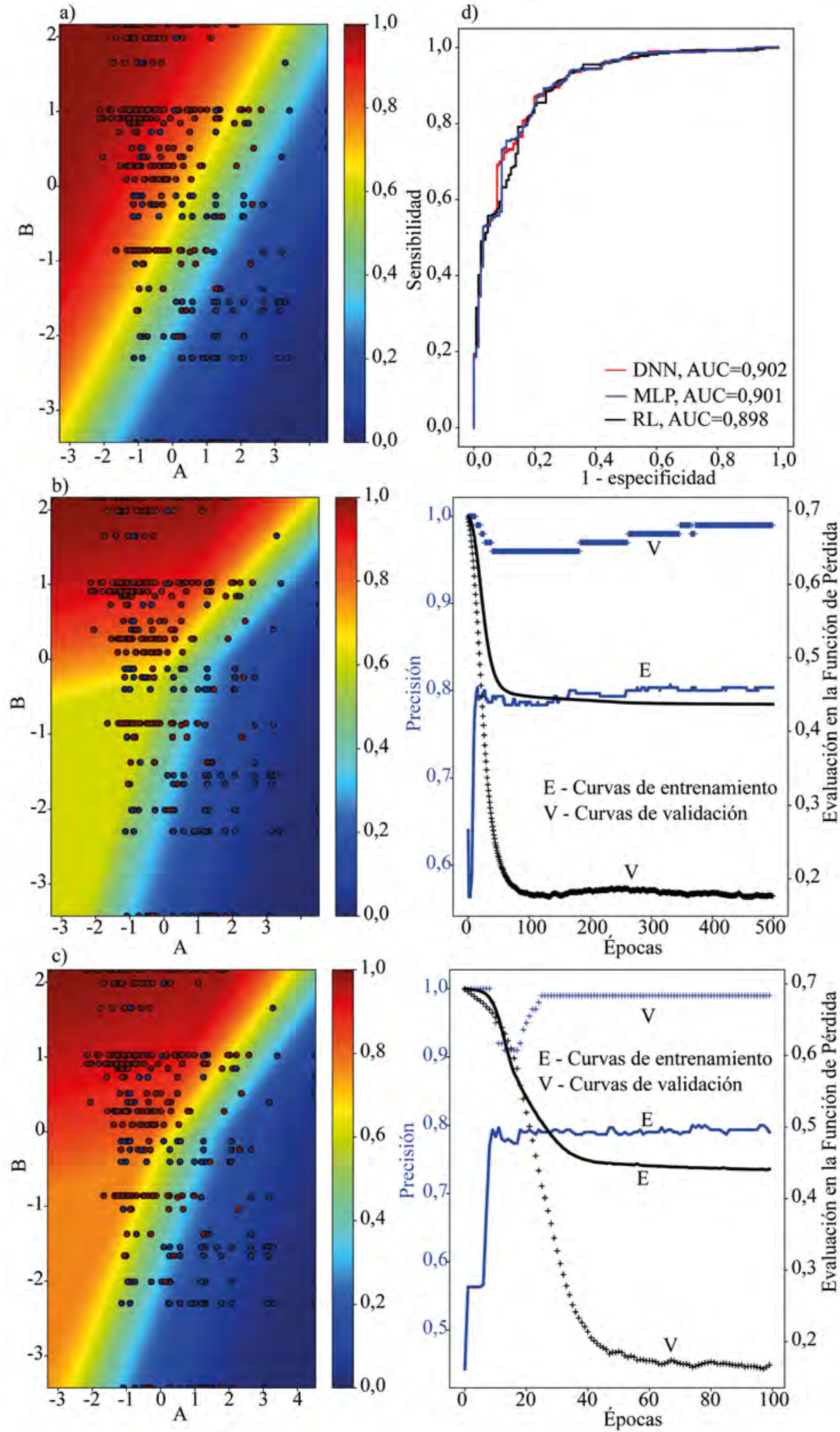

Figura 6. Evaluación de los modelos para la Propuesta 1 con (a) RL, (b) MLP, (c) DNN, y (d) su evaluación de predicción con las curvas ROC y AUC. 
En la Propuesta 2 se usaron como variables de entrada a K1, K2, K3, K4, K5, donde la frontera de decisión corresponde a hiperplanos que no son posibles de graficar debido a que tienen más de 5 dimensiones. La capacidad predictiva de cada modelo se evaluó a partir de las curvas ROC, como se muestra en la Figura 7a. Para RL se obtuvo el valor más bajo de $\mathrm{AUC}=$ 0,947 , y se verifica que las variables K2 y K3 tuvieron una significancia relativamente baja (ver Tabla 3), con $\mathbf{p}(>|\mathbf{z}|)>0,05$ o $|\mathbf{z}|<2$, lo que sugiere una baja capacidad predictiva en el modelo general RL. Por otra parte, la diferencia entre MLP y DNN es de 0,011 en los valores de AUC. En la Figura 7a y 7b se presentan los resultados de la precisión y evaluación de la función de pérdida durante el entrenamiento y, validación de las redes neuronales (MLP, DNN), respectivamente. Se observa un mejor desempeño del algoritmo de entrenamiento cuando se usan las 5 variables de entrada, llegando hasta una precisión cercana al 100\% para los datos de validación en apenas 100 y 45 épocas para MLP y DNN respectivamente.

Tabla 3. Resumen estadístico de RL para la Propuesta 2 con las variables K1, K2, K3, K4, K5.

\begin{tabular}{ccccc}
\hline & Coeficiente & Desviación estándar & $\mathbf{z}$ & $\mathbf{p}(>|\mathbf{z}|)$ \\
\hline K1 & 2,469 & 0,287 & 8,587 & 0,000 \\
K2 & 2,533 & $\mathbf{2 , 3 1 4}$ & $\mathbf{1 , 0 9 5}$ & $\mathbf{0 , 2 7 4}$ \\
K3 & $-0,269$ & 0,201 & $\mathbf{- 1 , 3 4 1}$ & $\mathbf{0 , 1 8 0}$ \\
K4 & 0,708 & 0,183 & 3,877 & 0,000 \\
K5 & 0,603 & 0,163 & 3,695 & 0,000 \\
Intercepto & 1,808 & 0,208 & 8,692 & 0,000 \\
\hline
\end{tabular}
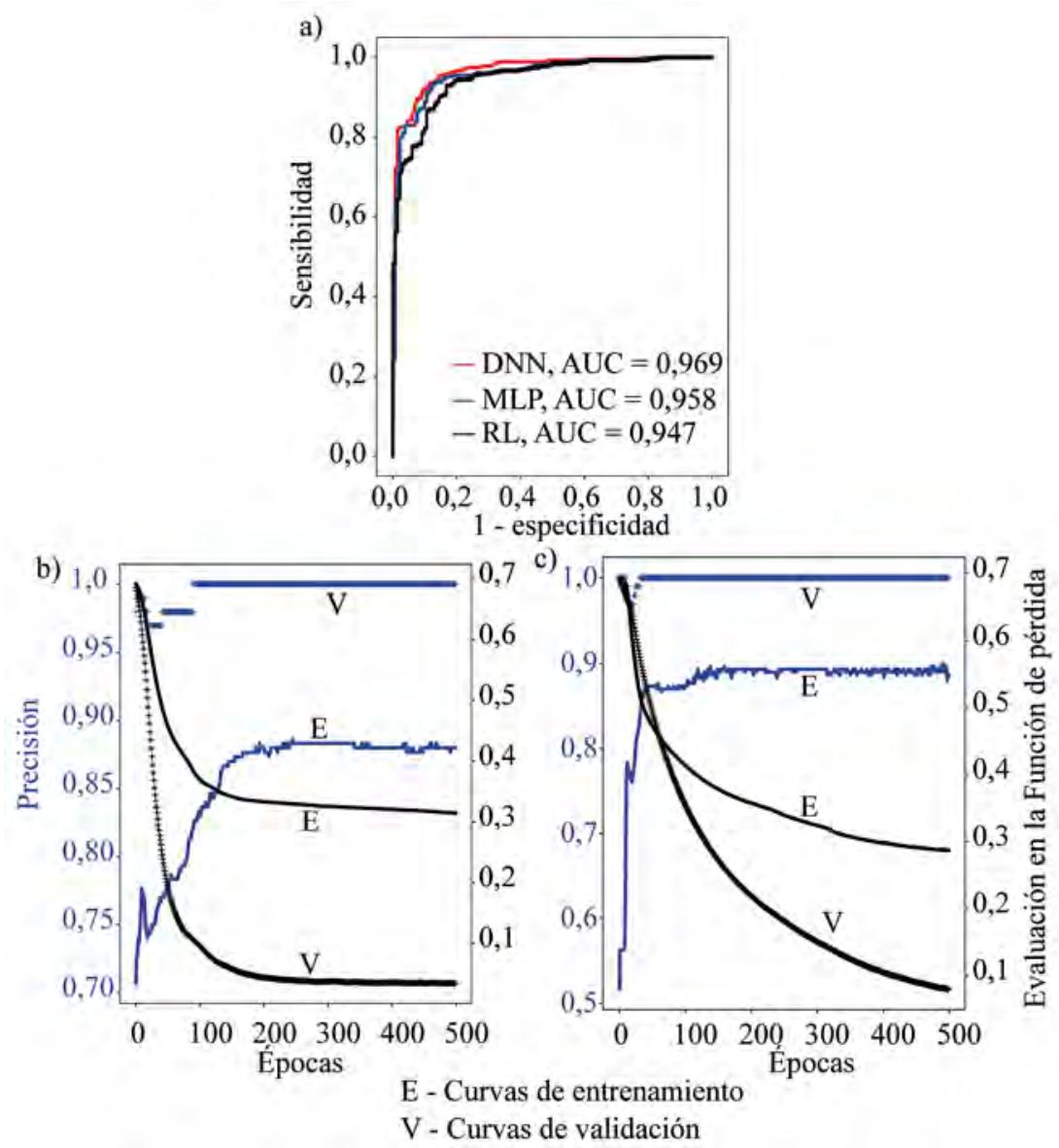

Figura 7. Evaluación de los modelos para la Propuesta 2. (a) Evaluación de la capacidad de predicción con las curvas ROC. Curvas de entrenamiento y validación para la precisión y Función de Pérdida en los modelos (b) MLP y (c) DNN. 


\section{Evaluación de la susceptibilidad}

Los mapas de susceptibilidad por movimientos en masa representan una manera gráfica de analizar visualmente las áreas propensas a deslizamientos (Wang et al., 2019). Por esta razón, es importante realizar una correcta cuantificación de la susceptibilidad con el objetivo de representar con la mayor precisión posible la distribución espacial de los datos. En la Figura 8 y Figura 9 se presentan los resultados obtenidos con la Propuesta 1 y la Propuesta 2, respectivamente, a través de los modelos RL, MLP y DNN. Adicionalmente, en la Figura 10 se presentan los resultados obtenidos con el método WoE, teniendo en cuenta los parámetros descritos por el Servicio Geológico Colombiano (2015), los cuales incluyen el uso de las unidades geológicas superficiales, la geomorfología, los usos del suelo y un inventario de deslizamientos.

La Figura 8 presenta los mapas de susceptibilidad por movimientos en masa obtenidos con la Propuesta 1. Los valores más altos de susceptibilidad se encuentran hacia el oeste y sureste de la zona de estudio y en el casco urbano del municipio, alcanzando valores superiores a 0,90 para los modelos RL y MLP, y siendo aproximadamente constantes $(0,86)$ para el modelo DNN. Hacia el oeste y sureste se presenta una alta densidad de deslizamientos como consecuencia de las condiciones geológicas y geomorfológicas de estas zonas, las cuales incluyen: una topografía con pendientes $>20^{\circ}$ en el oeste y de hasta $45^{\circ}$ en el sureste, geoformas de origen volcánico y estructural, $\mathrm{y}$ en menor proporción de origen denudacional (coluviones), suelos residuales poco cohesivos con presencia de pequeñas zonas de depósitos coluviales $\mathrm{y}$, en las zonas más escarpadas, rocas ígneas con poca meteorización (sector sureste). En el casco urbano del municipio la presencia de deslizamientos es mínima debido a una topografía plana con pendientes inferiores a $10^{\circ}$ localizadas en geoformas de abanico volcánico, sin embargo, los valores de susceptibilidad para los modelos RL y DNN son altos $(>0,90)$, aunque para el modelo MLP disminuyen levemente $(<0,75)$ como se logra observar en el círculo discontinuo de la Figura $8 \mathrm{~b}$ en comparación con la Figura 8a y 8c. Por otra parte, los valores intermedios $(0,60-0,80)$ y bajos $(<0,50)$ de susceptibilidad se encuentran en la parte central y oriental del municipio de Popayán, respectivamente. Hacia el centro se tiene una planicie de abanico volcánico con ausencia de deslizamientos, por el contrario, en el sector este del municipio, donde predominan las geoformas estructurales y volcánicas con pendientes superiores a $30^{\circ}$, hay una alta presencia de deslizamientos como se observa en el círculo continuo de la Figura 8, sin embargo, los valores de susceptibilidad calculados por los tres modelos son bajos para esta área.

La Figura 9 muestra los mapas de susceptibilidad por movimientos en masa obtenidos con la Propuesta 2. Los tres modelos presentan valores altos $(>0,90)$ de susceptibilidad hacia el este y noroeste del municipio, y bajos $(<0,10)$ hacia la parte central, abarcando el casco urbano, donde alrededor de este (excepto hacia el norte) se encuentran los valores intermedios de susceptibilidad. A pesar de que los tres mapas presentan características similares, el modelo DNN correspondiente a la Figura 9c, presenta áreas mucho más homogeneizadas en las zonas topográficamente planas como los abanicos volcánicos y en las zonas de pendientes escarpadas localizadas al este del municipio, a diferencia de los modelos RL y MLP mostrados en la Figura 9a y 9b, respectivamente. Adicionalmente, los depósitos coluviales se encuentran mejor identificados y con valores de susceptibilidad más altos en el modelo DNN en relación con los demás, como se señala en el círculo discontinuo de la Figura 9. Con respecto a la Figura 8, las mayores diferencias se observan hacia el sector más oriental del área de estudio (círculo continuo), donde hay deslizamientos cartografiados que no fueron identificados por ninguno de los modelos en la Propuesta 1 en comparación con la Propuesta 2, donde los tres modelos presentan susceptibilidad alta en esta zona, como se señala en el círculo continuo de la Figura 9. Además, la parte central del municipio y su casco urbano presentan valores muy bajos $(<0,10)$ de susceptibilidad en la Propuesta 2, en contraste con la Propuesta 1 que presenta valores intermedios en esta zona $(>0,50)$, lo cual no es acorde con el inventario de deslizamientos debido a que este señala una ausencia de eventos en esta área.

Finalmente, en la Figura 10 se muestra el mapa de susceptibilidad obtenido con el método WoE. Al igual que los mapas de la Figura 8 y Figura 9, también es posible identificar que hacia la periferia del municipio de Popayán (sector oeste y sureste) se presentan los valores más altos de susceptibilidad y que, hacia la parte central y en el casco urbano, se encuentran los valores más bajos. No obstante, similar a los resultados obtenidos con la Propuesta 1, el método de WoE no identifica con susceptibilidad alta el sector más oriental de la zona de estudio, como se señala en el círculo continuo de la Figura 10, a pesar de que los factores condicionantes e inventario indican una alta probabilidad de deslizamientos en esa área. 

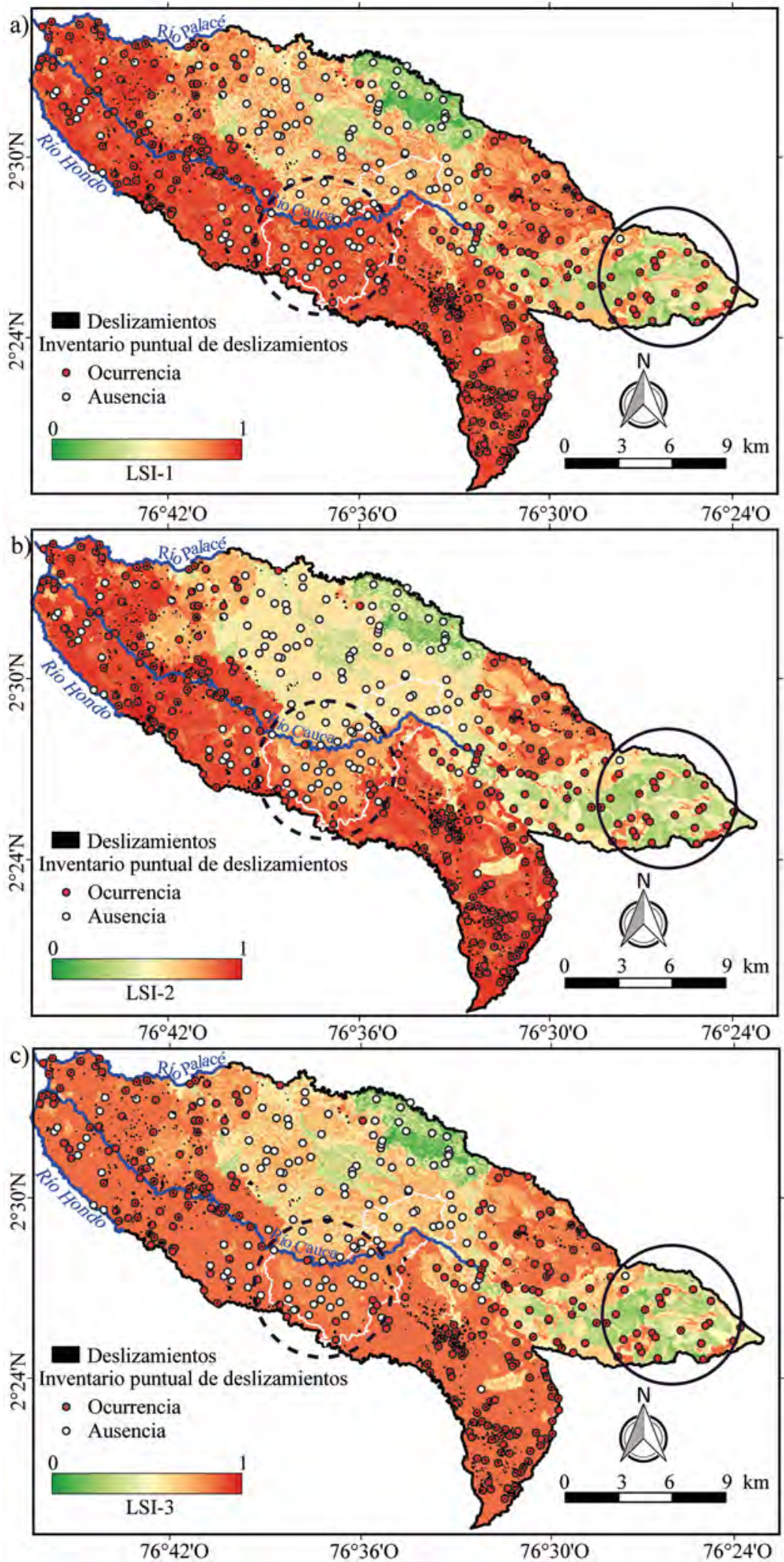

Figura 8. Mapas de susceptibilidad por movimientos en masa para la Propuesta 1: (a) Modelo RL; (b) Modelo MLP; (c) Modelo DNN. El círculo discontinuo indica la zona con mayor diferencia entre los tres modelos, mientras que el círculo continuo indica la zona con mayor diferencia con respecto a la Propuesta 2. 

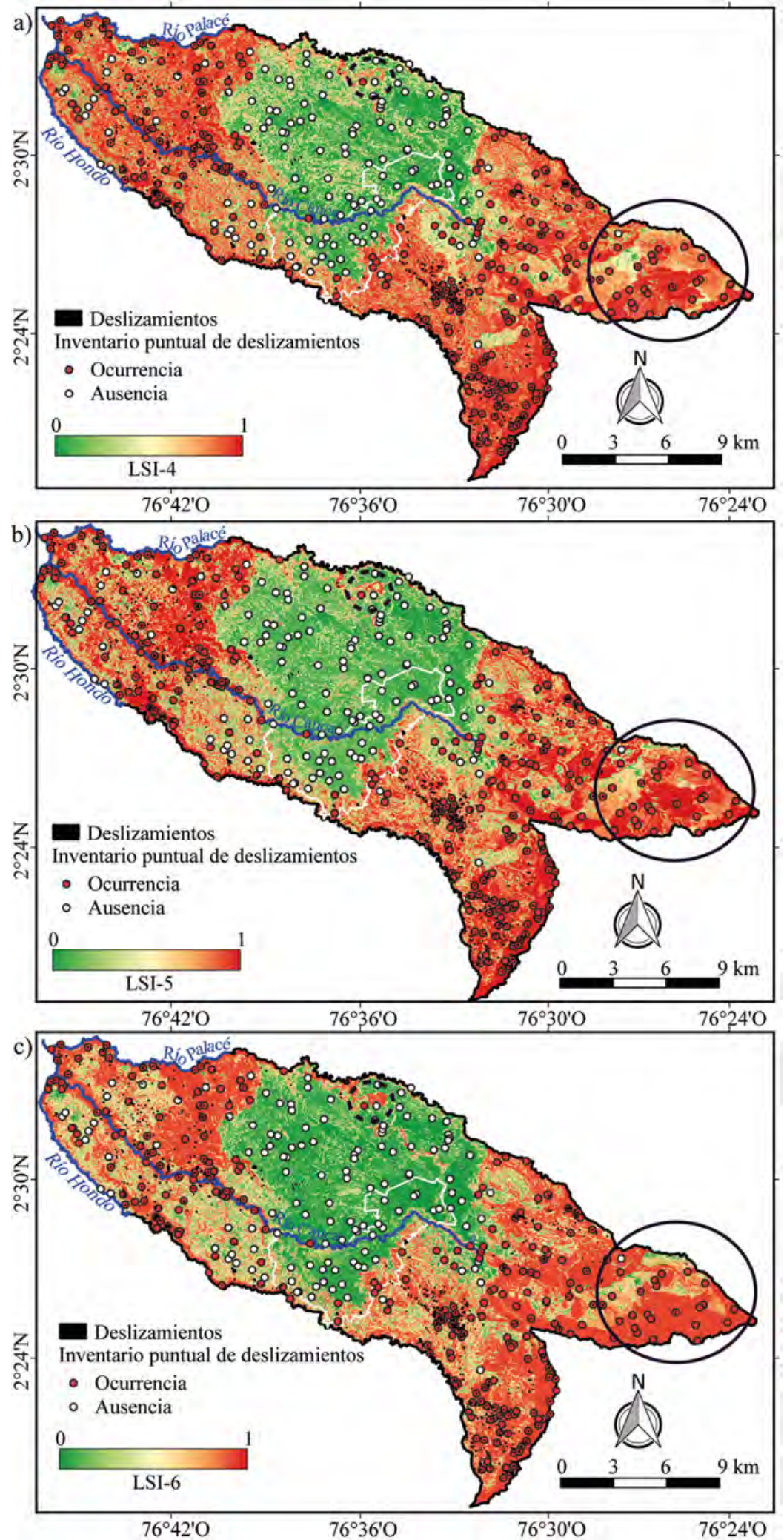

Figura 9. Mapas de susceptibilidad por movimientos en masa para la Propuesta 2: (a) Modelo RL; (b) Modelo MLP; (c) Modelo DNN. El círculo discontinuo señala los depósitos coluviales, mientras que el círculo continuo señala la zona con mayor diferencia con respecto a la Propuesta 1 y el método WoE. 


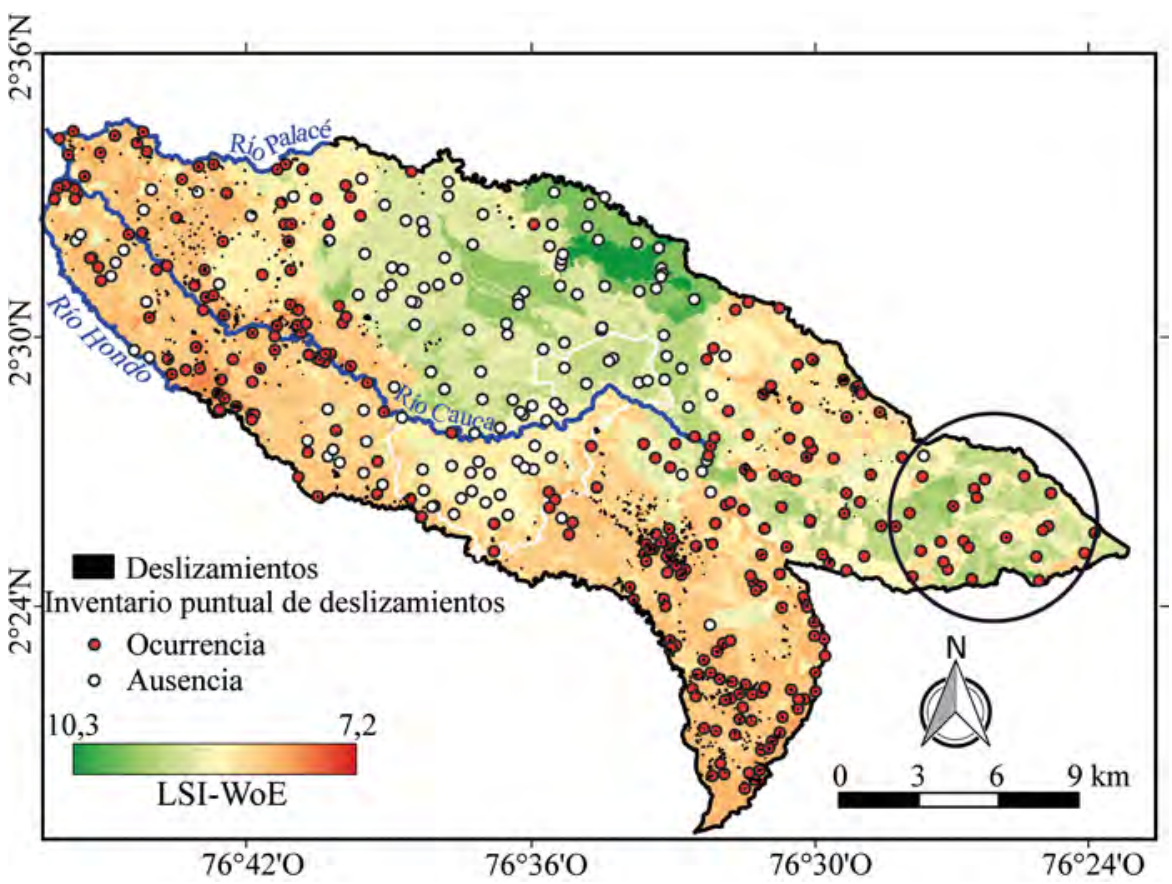

Figura 10. Mapa de susceptibilidad por movimientos en masa elaborado con el método WoE, según la metodología del Servicio Geológico Colombiano. El círculo continuo indica la zona con mayor diferencia con respecto a la Propuesta 2.

\section{Conclusiones}

En este estudio se desarrollaron dos propuestas para el cálculo de la susceptibilidad por movimientos en masa a partir un modelo de doble evaluación utilizando WoE y ANN. Para ANN se evaluó la función de pérdida del tipo entropía cruzada binaria, la cual es adecuada y coherente con la naturaleza de los datos del inventario de deslizamientos, por lo que la red neuronal tiene mejor capacidad de predicción al encontrar las relaciones no lineales entre los factores condicionantes y los valores del entrenamiento.

Las dos propuestas desarrolladas fueron evaluadas con el valor de AUC de sus respectivas curvas ROC, donde la Propuesta 2 con valores $\mathrm{AUC}=0,947$ (RL), 0,958 (MLP) y 0,969 (DNN) obtuvo un mejor desempeño en comparación con la Propuesta 1 con valores de $\mathrm{AUC}=$ 0,898 (RL), 0,901 (MLP) y 0,902 (DNN). Además, teniendo en cuenta los valores de AUC, se verifica que el método con una mayor capacidad de predicción corresponde al DNN tanto para la Propuesta 1 como para la Propuesta 2.

Por otra parte, los mapas de susceptibilidad por movimientos en masa obtenidos con las dos propuestas reflejan importantes diferencias entre ellas. Para la Propuesta 1 , se observa que en los tres modelos la parte central del municipio y su casco urbano presentan valores altos de susceptibilidad, mientras que el sector oriental presenta valores bajos. Por el contrario, los resultados obtenidos con la Propuesta 2 muestran que los valores más bajos de susceptibilidad se encuentran hacia el centro del municipio y los más altos hacia el sector oriental, lo cual representa la realidad en cuanto a ocurrencia de deslizamientos. Además, el modelo DNN en la Propuesta 2 homogeniza con mayor precisión estas áreas y resalta de manera más eficiente los depósitos coluviales.

Teniendo en cuenta lo anterior, el uso de DNN para la evaluación de la susceptibilidad por movimientos en masa en la Propuesta 2 representa una alternativa precisa en la predicción espacial de deslizamientos. Además, los mapas de susceptibilidad obtenidos a partir de este modelo podrían representar una evaluación más detallada a escala 1:25000 o superior para los proyectos de planeación municipal, con el objetivo de optimizar la delimitación de los sectores prioritarios en los que se deberán proponer estudios detallados.

\section{Agradecimientos}

Los autores le agradecen al Servicio Geológico Colombiano por proporcionar los datos e información usada en este trabajo, y a Rosana Báez Rodríguez 
por sus comentarios realizados sobre la evaluación de la susceptibilidad con métodos cuantitativos. Los revisores proporcionaron amplios comentarios y sugerencias que mejoraron sustancialmente el manuscrito.

\section{Repositorio y acceso a datos}

Los datos usados en este trabajo están protegidos bajo una licencia de acceso libre y se encuentran publicados en la plataforma web miig.sgc.gov.co. Este trabajo fue elaborado en su totalidad usando código abierto y programas libres como Python, QGIS y pyQGIS. En el siguiente repositorio se pueden encontrar los códigos en Python 3x, así como los insumos geográficos usados https://github.com/PAULGOYES/Landslide_ RL_MLP_DNN.

\section{Referencias}

Abbaszadeh-Shahri, A.; Spross, J.; Johansson, F.; Larsson, S. (2019). Landslide susceptibility hazard map in southwest Sweden using artificial neural network. Catena, 183. https://doi.org/10.1016/j. catena.2019.104225

Aditian, A.; Kubota, T.; Shinohara, Y. (2018). Comparison of GIS-based landslide susceptibility models using frequency ratio, logistic regression, and artificial neural network in a tertiary region of Ambon, Indonesia. Geomorphology, 318, 101-111. https://doi.org/10.1016/j.geomorph.2018.06.006

Aristizábal, E.; López, S.; Sánchez, O.; Vásquez, M.; Rincón, F.; Ruiz-Vásquez, D.; Restrepo, S.; Valencia, J.S. (2019). Evaluación de la amenaza por movimientos en masa detonados por lluvias para una región de los Andes colombianos estimando la probabilidad espacial, temporal, y magnitud. Boletín de Geología, 41(3), 85-105. https://doi.org/10.18273/revbol.v41n3-2019004

Asf-Alaska. (2007). Dataset: (C) JAXA/METI ALOS PALSAR L1.0 2007. Recuperado de ASF DAAC. Consultado el 11 de junio de 2020. https://doi. org/10.5067/NXY378J3DFZQ

Baeza, C.; Corominas, J. (2001). Assessment of shallow landslide susceptibility by means of multivariate statistical techniques. Earth Surface
Processes and Landforms, 26(12), 1251-1263. https://doi.org/10.1002/esp.263

Bragagnolo, L.; da Silva, R.V.; Grzybowski, J.M.V. (2020). Landslide susceptibility mapping with r.landslide: A free open-source GIS-integrated tool based on Artificial Neural Networks. Environmental Modelling and Software, 123. https://doi.org/10.1016/j.envsoft.2019.104565

Brownlee, J. (2017). Gentle Introduction to the Adam Optimization Algorithm for Deep Learning. Deep Learning Performance. https://machinelearningmastery.com/adamoptimization-algorithm-for-deep-learning/

Bui, D.T.; Tuan, T.A.; Klempe, H.; Pradhan, B.; Revhaug, I. (2016). Spatial prediction models for shallow landslide hazards: a comparative assessment of the efficacy of support vector machines, artificial neural networks, kernel logistic regression, and logistic model tree. Landslides, 13(2), 361-378. https://doi.org/10.1007/s10346015-0557-6

Bui, D.T.; Tsangaratos, P.; Nguyen, V.T.; Van Liem, N.; Trinh, P.T. (2020). Comparing the prediction performance of a Deep Learning Neural Network model with conventional machine learning models in landslide susceptibility assessment. Catena, $188 . \quad$ https://doi.org/10.1016/j. catena.2019.104426

Cantarino, I.; Carrion, M.A.; Goerlich, F.; MartinezIbañez, V. (2019). A ROC analysis-based classification method for landslide susceptibility maps. Landslides, 16(2), 265-282. https://doi. org/10.1007/s10346-018-1063-4

Carvajal-Perico, J.H. (2012). Propuesta de estandarización de la cartografía geomorfológica en Colombia. Bogotá: Servicio Geológico Colombiano.

Chollet, F. (2018). Deep Learning with Python. Manning.

Corominas, J.; van Westen, C.; Frattini, P.; Cascini, L.; Malet, J.P.; Fotopoulou, S.; Catani, F.; Van Den Eeckhaut, M.; Mavrouli, O.; Agliardi, F.; Pitilakis, K.; Winter, M.G.; Pastor, M.; Ferlisi, S.; Tofani, V.; Hervás, J.; Smith, J.T. (2014). 
Recommendations for the quantitative analysis of landslide risk. Bulletin of Engineering Geology and the Environment, 73(2), 209-263. https://doi. org/10.1007/s10064-013-0538-8

Correa, F.; Gallardo, J.; Muñoz, N.; Pérez, R. (2016). Estudio comparativo basado en métricas para diferentes arquitecturas de navegación reactiva. Ingeniare, 24(1), 46-54. https://doi.org/10.4067/ S0718-33052016000100005

Correa-Muñoz, N.A.; Higidio-Castro, J.F. (2017). Determination of landslide susceptibility in linear infrastructure. Case: aqueduct network in Palacé, Popayan (Colombia). Ingeniería e Investigación, $37(2), \quad 17-24$. https://doi.org/10.15446/ing. investig.v37n 2.59654

Dai, F.C.; Lee, C.F.; Ngai, Y.Y. (2002). Landslide risk assessment and management: an overview. Engineering Geology, 64(1), 65-87. https://doi. org/10.1016/S0013-7952(01)00093-X

DANE. (2019). Resultados Censo Nacional de Población y Vivienda 2018. Consultado el 18 de marzo de 2020. http://microdatos.dane.gov.co/ index.php/catalog/643

Decreto 1077 de 2015 [con fuerza de ley]. Por medio del cual se expide el Decreto Único Reglamentario del Sector Vivienda, Ciudad y Territorio. 26 de mayo de 2015. D.O. No. 49523.

Goodfellow, I.; Bengio, Y.; Courville, A. (2016). Deep Learning. MIT Press.

Gwelo, A. (2019). Principal components to overcome multicollinearity problem. Oradea Journal of Business and Economics, 4(1), 79-91.

He, Q.; Shahabi, H.; Shirzadi, A.; Li, S.; Chen, W.; Wang, N.; Chai, H.; Bian, H.; Ma, J.; Chen, Y.; Wang, X.; Chapi, K.; Ahmad, B.B. (2019). Landslide spatial modelling using novel bivariate statistical based Naïve Bayes, RBF Classifier, and RBF Network machine learning algorithms. Science of the Total Environment, 663, 1-15. https://doi.org/10.1016/j.scitotenv.2019.01.329

Hemasinghe, H.; Rangali, R.S.S.; Deshapriya, N.L.; Samarakoon, L. (2018). Landslide susceptibility mapping using logistic regression model (a case study in Badulla District, Sri Lanka). Procedia
Engineering, 212, 1046-1053. https://doi. org/10.1016/j.proeng.2018.01.135

Hong, H.; Ilia, I.; Tsangaratos, P.; Chen, W.; Xu, C. (2017). A hybrid fuzzy weight of evidence method in landslide susceptibility analysis on the Wuyuan area, China. Geomorphology, 290, 1-16. https:// doi.org/10.1016/j.geomorph.2017.04.002

Hu, Q.; Zhou, Y.; Wang, S.; Wang, F. (2020). Machine learning and fractal theory models for landslide susceptibility mapping: Case study from the Jinsha River Basin. Geomorphology, 351. https:// doi.org/10.1016/j.geomorph.2019.106975

Huang, F.; Zhang, J.; Zhou, C.; Huang J.; Zhu L. (2020). A deep learning algorithm using a fully connected sparse autoencoder neural network for landslide susceptibility prediction. Landslides, 17, 217-229. https://doi.org/10.1007/s10346-01901274-9

Ilia, I.; Tsangaratos, P.; Koumantakis, I.; Rozos, D. (2010). Application of a bayesian approach in GIS based model for evaluating landslide susceptibility. Case study KIMI area, Euboea, Greece. Bulletin of the Geological Society of Greece, 43(3), 15901600. https://doi.org/10.12681/bgsg.11333

Ilia, I.; Tsangaratos, P. (2016). Applying weight of evidence method and sensitivity analysis to produce a landslide susceptibility map. Landslides, 13(2), 379-397. https://doi.org/10.1007/s10346015-0576-3

Kadavi, P.R.; Lee, C.W.; Lee, S. (2019). Landslidesusceptibility mapping in Gangwon-do, South Korea, using logistic regression and decision tree models. Environmental Earth Sciences, 78(4). https://doi.org/10.1007/s12665-019-8119-1

Kingma, D.P.; Ba, J.L. (2015). Adam: A method for stochastic optimization. 3rd International Conference on Learning Representations. San Diego, EE.UU.

Lei, T.C.; Wan, S.; Chou, T.Y.; Pai, H.C. (2011). The knowledge expression on debris flow potential analysis through PCA + LDA and rough sets theory: A case study of Chen-Yu-Lan watershed, Nantou, Taiwan. Environmental Earth Sciences, 63(5), 981-997. https://doi.org/10.1007/s12665010-0775-0 
Lin, G.F.; Chang, M.J.; Huang, Y.C.; Ho, J.Y. (2017). Assessment of susceptibility to rainfall-induced landslides using improved self-organizing linear output map, support vector machine, and logistic regression. Engineering Geology, 224, 62-74. https://doi.org/10.1016/j.enggeo.2017.05.009

Lombardo, L.; Mai, P.M. (2018). Presenting logistic regression-based landslide susceptibility results. Engineering Geology, 244, 14-24. https://doi. org/10.1016/j.enggeo.2018.07.019

Luo, W.; Liu, C.C. (2018). Innovative landslide susceptibility mapping supported by geomorphon and geographical detector methods. Landslides, 15(3), 465-474. https://doi.org/10.1007/s10346017-0893-9

Mahdadi, F.; Boumezbeur, A.; Hadji, R.; Kanungo, D.P.; Zahri, F. (2018). GIS-based landslide susceptibility assessment using statistical models: a case study from Souk Ahras province, N-E Algeria. Arabian Journal of Geosciences, 11(17). https://doi.org/10.1007/s12517-018-3770-5

Oh, H.J.; Kadavi, P.R.; Lee, C.W.; Lee, S. (2018). Evaluation of landslide susceptibility mapping by evidential belief function, logistic regression and support vector machine models. Geomatics, Natural Hazards and Risk, 9(1), 1053-1070. https://doi.org/10.1080/19475705.2018.1481147

Ozdemir, A.; Altural, T. (2013). A comparative study of frequency ratio, weights of evidence and logistic regression methods for landslide susceptibility mapping: Sultan Mountains, SW Turkey. Journal of Asian Earth Sciences, 64, 180-197. https://doi. org/10.1016/j.jseaes.2012.12.014

Pamela; Sadisun, I.A.; Arifianti, Y. (2018). Weights of evidence method for landslide susceptibility mapping in Takengon, Central Aceh, Indonesia. IOP Conference Series: Earth and Environmental Science, 118(1). https://doi.org/10.1088/17551315/118/1/012037

Patriche, C.V.; Pirnau, R.; Grozavu, A.; Rosca, B. (2016). A comparative analysis of binary logistic regression and analytical hierarchy process for landslide susceptibility assessment in the Dobrovăț River Basin, Romania. Pedosphere, 26(3), 335-350. https://doi.org/10.1016/S10020160(15)60047-9
Regmi, N.R.; Giardino, J.R.; Vitek, J.D. (2010). Modeling susceptibility to landslides using the weight of evidence approach: Western Colorado, USA. Geomorphology, 115(1-2), 172-187. https:// doi.org/10.1016/j.geomorph.2009.10.002

Sagar, B.S.D.; Cheng, Q.; Agterberg, F. (2018). Handbook of Mathematical Geosciences: Fifty Years of IAMG. Springer International Publishing. https://doi.org/10.1007/978-3-319-78999-6

Servicio Geológico Colombiano. (2015). Zonificación geomecánica y de amenaza por movimientos en masa del municipio de Popayán - Cauca.

Servicio Geológico Colombiano. (2017). Guía Metodológica para la Zonificación de Amenaza por Movimientos en Masa Escala 1:25000.

Tharwat, A. (2020). Classification assessment methods. Applied Computing and Informatics. https://doi. org/10.1016/j.aci.2018.08.003

Trigila, A.; Iadanza, C.; Esposito, C.; ScarasciaMugnozza, G. (2015). Comparison of Logistic Regression and Random Forests techniques for shallow landslide susceptibility assessment in Giampilieri (NE Sicily, Italy). Geomorphology, 249, 119-136. https://doi.org/10.1016/j. geomorph.2015.06.001

van Westen, C.J. (2002). Weights of evidence modeling for landslide susceptibility mapping. International Institute for Geoinformation Science and Earth Observation (ITC), Netherlands.

van Westen, C.J.; Rengers, N.; Soeters, R. (2003). Use of geomorphological information in indirect landslide susceptibility assessment. Natural Hazards, 30(3), 399-419. https://doi.org/10.1023/ B:NHAZ.0000007097.42735.9e

van Westen, C.J.; Castellanos, E.; Kuriakose, S.L. (2008). Spatial data for landslide susceptibility, hazard, and vulnerability assessment: An overview. Engineering Geology, 102(3-4), 112-131. https:// doi.org/10.1016/j.enggeo.2008.03.010

Varnes, D. (1978). Slope movement types and processes. En: R. Schuster; R. Krizek (eds.). Landslides: analysis and control (pp. 11-33). Transportation Research Board. 
Wang, Y.; Fang, Z.; Hong, H. (2019). Comparison of convolutional neural networks for landslide susceptibility mapping in Yanshan County, China. Science of the Total Environment, 666, 975-993. https://doi.org/10.1016/j.scitotenv.2019.02.263

Yang, J.; Song, C.; Yang, Y.; Xu, C.; Guo, F.; Xie, L. (2019). New method for landslide susceptibility mapping supported by spatial logistic regression and GeoDetector: A case study of Duwen Highway Basin, Sichuan Province, China. Geomorphology, 324, 62-71. https://doi. org/10.1016/j.geomorph.2018.09.019

Zhao, Y.; Wang, R.; Jiang, Y.; Liu, H.; Wei, Z. (2019). GIS-based logistic regression for rainfall- induced landslide susceptibility mapping under different grid sizes in Yueqing, Southeastern China. Engineering Geology, 259. https://doi. org/10.1016/j.enggeo.2019.105147

Zhou, C.; Yin, K.; Cao, Y.; Ahmed, B.; Li, Y.; Catani, F.; Pourghasemi, H.R. (2018). Landslide susceptibility modeling applying machine learning methods: A case study from Longju in the Three Gorges Reservoir area, China. Computers \& Geosciences, 112, 23-37. https:// doi.org/10.1016/j.cageo.2017.11.019

Fecha de recibido: 01 de abril de 2020

Fecha de aprobado: 21 de octubre de 2020 